\title{
A Semi-supervised Puzzle-Based Method for Separating the Venous and Arterial Vascular Networks in Retinal Images
}

\author{
Edgardo M. Felipe-Riveron ${ }^{1}$, Fabiola M. Villalobos Castaldi ${ }^{2}$, \\ Ernesto Suaste Gómez ${ }^{2}$, Marcos A. Leiva Vasconcellos ${ }^{1}$, \\ and Cecilia Albortante Morato ${ }^{3}$ \\ ${ }^{1}$ Center for Computing Research, National Polytechnic Institute, Av. Juan de Dios Batiz and \\ Miguel Othon de Mendizabal P.O. 07738, \\ Mexico D. F., Mexico \\ edgardo@cic.cin.mx, shadowalker25403@yahoo.com \\ ${ }^{2}$ Researches and Advanced Studies Center of the National Polytechnic Institute, \\ Av. Instituto Politécnico Nacional \# 2508, La Laguna Ticoman, Gustavo A. Madero, \\ P.O. 07360, Mexico D.F., Mexico \\ \{mvillalobos, esuaste\} @cinvestav.mx, \\ ${ }^{3}$ University of Camagüey, Carretera Circunvalación Norte Km 51/2, Camagüey, Cuba \\ cecitog@yahoo.com.mx
}

\begin{abstract}
The focus of this work is to create a methodology to separate the entire vascular network into its independent veins and arteries networks in optical human fundus images. It has been developed following the logical procedure used by humans when they assemble a puzzle. In the development of the methodology we take into consideration physiological properties, topological properties of the tree structure and morphological properties of both networks, that is, they have only bifurcations, crosses and ending points, and also that crosses are produced always between venous and arterial branches. For arterial blood vessels we get a classification capability, based on the pixel counting, of $84.88 \%$ while for venous was $82.87 \%$. This indicates that the methodology classified correctly as average $83.80 \%$ of the total blood vessels in the images.
\end{abstract}

Keywords: Retinal image analysis, retinal vascular network separation, puzzle-based method.

\section{$1 \quad$ Introduction}

The human retina is mainly conformed anatomically by the optic disc (OD), the macula luteal and the arterial and venous vascular networks. Arteries transport blood with high oxygen content to the organs and veins bring the oxygen-poor blood back to the heart-lung circulation. The separation of the retinal vascular network is a complex task. It can present some difficulties because frequently one of the networks obstructs the visibility of the other one, leading to a serious confusion between them. However, a good splitting of the vein and arterial networks allows the detection of some structural characteristics and functionalities that hides when the network is analyzed as a whole. 
An important aspect to make a good vessel network splitting is to take into consideration the topology of the tree structure and the morphological properties of both networks. An ophthalmologist is able to track the venous and arterial networks, but when they have too many branches, it demands to track more than once each network in order to analyze the entire vessels.

The analysis of the vascular network by an ophthalmologist to make an accurate diagnosis will be easier and better if he/she have two images with the veins and arterial networks separated. The situation is the same, or maybe more justified, if our intention consists of analyze by a computer automatically the quality of the retinal venous and arterial networks. Undoubtedly, the analysis will be easier if both networks are completely independent. Some common analysis of the retinal vascular network could be related with the detection of stenosis, aneurysms, hemorrhages, thrombus, tortuosity, and many other conditions that could indicate that the vascular network is not healthy. It is taken into consideration for the development of the proposed methodology the important physiological property that arteries and veins never intersect between themselves neither creates loops, although a vein and an artery can concur forming an artery-vein joined trajectory.

The focus of this work is to create a methodology to separate the entire vascular network into its independent veins and arteries networks in human fundus images. The methodology has been developed following the logical procedure commonly used by humans when they assemble a puzzle.

\section{Antecedents}

There is a lot of works about blood vessel network segmentation in the literature. However, works related to the separation of vascular blood vessels networks in its venous and arterial branches has received a limited attention so far. Computerized analysis of retinal fundus images potentially reduce the workload of ophthalmologists and can improve in many ways the diagnostic efficiency, mainly when it is related to the quality of the patient's retinal vascular network [1-8, 13]. Its clinical status can say too much to physicians about the general status of the vascular network located in other zones in the human body. Besides this, particularly the retinal vascular network is affected with very dangerous results (blindness) with diabetes (diabetic retinopathy), high blood pressure (hypertensive retinopathy), and other common systemic diseases.

The automated classification of blood vessels in the retina as venous and arterials is still an open problem in image processing. In a fundus image veins and arteries differ visually in their shapes, thickness, color and texture. These differences can be easily appreciated in main vessels near to the optic disc, but varies strongly in vessels more far from the optic disk. That is one of the reasons that the separation of the vascular network of the retina cannot be done successfully under these bases.

\section{The Proposed Method}

The proposed methodology for splitting the retinal vascular network in their corresponding venous and arterial branches follows the steps indicated in the flow diagram shown in figure 1 . In this paper are described only the last three steps (from step 4 to 
6). This means that we start the procedure from images having the vascular network already segmented. For the interpretation of the references to color in this study, the reader is referred to the web version of the paper.

The algorithm is based on the analysis of adjacent cells appearing each time when the tracking through a given blood vessel is carried out. In this task is taken into account the situation occurring between the number of independent blood elements contained in each cell and the number of contacts that the branches of the vascular network makes with the sides of the corresponding cell. It is semi-supervised because at this stage of the work we have not solved yet the automatic initial classification in venous and arterial the cells bordering the optic disc. Currently this is done manually before running the program to separate the venous and arterial vascular networks, which is fully non-supervised (automatic). First of all, a rectangular (or square) regular grid is superimposed over the image before the beginning of the separation of the vascular network in its arterial and venous branches, regardless whether the image was rotated, translated or scaled. Therefore, it is not necessary to consider the amounts of translation, rotation or scaling. The separation of the networks will take place regardless of the conditions of the retina image to which the method is facing. Ten different images with well-segmented vascular network were more than enough to tune the separation algorithm and prove its effectiveness.

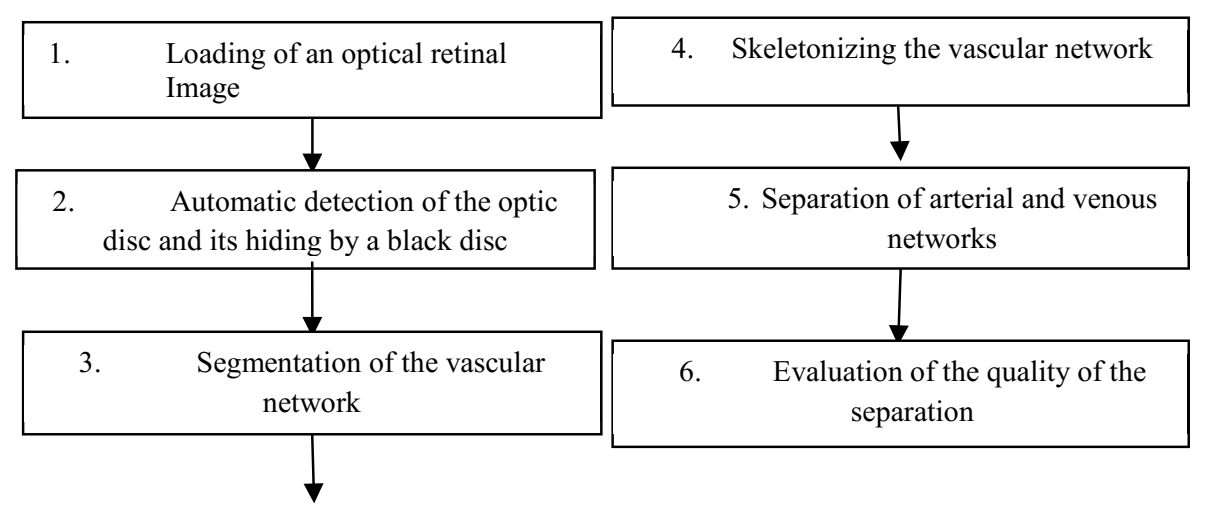

Fig. 1. Steps for separating the vascular networks of an optical retinal image

\subsection{Retinal Image Databases}

Since it is not a particular goal of our work to segment the vascular network, the system uses the corresponding segmented image from the DRIVE database, having the blood vessels visible at least until the second bifurcation. This means that the proposed methodology was developed and evaluated by using the set of 40 color fundus images of the training and test sets in the publicly available DRIVE database [9], [10].

We also worked with the images from the ARIA database [11]. Images with the segmented vascular network can also come from other systems using any algorithm or methodology reported in the literature for that task, if they provide good results and fulfill with the condition that branches of the network are clearly visible at least until the second bifurcation. 


\subsubsection{Physiological, Morphological and Morphometric Characteristics to Take into Consideration for the Separation of the Vascular Network}

The main physiological, morphological and morphometric characteristics that distinguishes the vascular networks are:

- Both branches (venous and arterial) of the vascular network present a tree structure.

- The main topological characteristic of each type of network is the presence of bifurcations. Sometime could appear trifurcations which are transformed in two consecutive bifurcations.

- Arterial blood always enters the retina and venous blood leaves the retina.

- Through any of the branches of any vascular network the blood flows backward. Bifurcations cannot have an angle between branches greater than 90 degree.

- The main arterial branch is called central retinal artery and the main venous branch is called central retinal vein.

- Both branches dichotomize in nasal and temporal on each eye and then dichotomize again into upper and lower branches.

- In areas of the macula luteal are not appreciated either veins or arteries. Therefore, the fovea, located at the center of the macula, is avascular.

- The zone where the optic disc appears in the fundus image is the nasal zone. The macula luteal of each eye is located at the opposite side.

- Between two consecutive bifurcations, or between a bifurcation and a visible end of a vein or artery, appears correspondingly a single venous or arterial branch.

- In a normal retina, branches between two consecutive bifurcations always have the same thickness, i.e. the thickness of the branch is constant.

- The locations of venous or arterial bifurcations have not any relation with the locations of bifurcations of the other network.

- The primary, secondary and tertiary arterial and venous branches are the most interesting. In a primary branch occur the first bifurcations; in a secondary branch occur the second bifurcations and in a tertiary branch occur the third bifurcation. A bifurcation always divides a vessel into two thinner vessels.

- The branches of the arterial and venous networks at any level interbreed with a branch of another type. Crossings do not divide the branches. The crosses are always between venous and arterial branches. The crossing branches can be between arteries or veins of primary, secondary or tertiary level. The different branches of the arterial and venous networks may have crossings anywhere in the retina, except on the macular zone. The branches of the venous network normally travel below the arterial network.

- Both the veins and arteries in a normal network have tubular structure. We consider as circular the cross section of veins and arteries.

\subsubsection{Mesh Creation}

Since the separation in the venous and arterial networks was carried out by local analysis within cells in a square lattice superimposed over the original segmented image, the size of the square cell considered suitable for the study was $20 \times 20$ pixels. This 
size should ensure that the cells could contain a segment (or more than one) of a vein or of an artery, a branch of one kind or another, crosses between vessels of different types, or an ending point of either type [12]. This cell size constituted the "puzzle piece" of the proposed methodology.

With this size of $20 \times 20$ pixels the percent of cells that will not be analyzed is less than $1 \%$ in images with a previously hidden optic disc. The number of key cells with crosses between veins and arteries, and arterial or venous bifurcations or trifurcations remained with an acceptable frequency of occurrence of about $37 \%$. The rest was occupied by single segments of veins or arteries, endpoints of one kind or another, or simply they were empty.

\subsubsection{Methodology for the Separation of the Vascular Network}

Although in this paper the separation process starts from a binary image with the vascular network already segmented, the complete methodology to separate the venous and arterial vascular networks covers the execution of the following procedures in the strict order indicated:

1. Detection of the optic disc.

2. Hiding of the optic disc with a black disc

3. Splitting the image of the segmented vascular network superimposing a grid (or mesh) of disjoint square cells of size 20 x 20 pixels.

4. Once the vessels around the optic disc have been manually classified as arterial or venous, explore the network starting from any of the previously identified cells surrounding the optic disc.

5. Determine every time the number of contacts of vessels inside the cell with its four edges and the number of connected components inside it.

6. With this information and the prior knowledge of the type of vessel(s) inside the current cell, and depending on the result of the analysis described in previous step 5 , it is possible to determine if the type of network in the next cell in any direction (North, South, West or East) is a simple branch (venous or arterial); or a bifurcation (venous or arterial); or a cross between a venous and an arterial segments; or an ending point (arterial or venous); or simply an empty cell.

7. Based on the decision of the previous step 6 , to store the necessary data to tabulate the group of related pixels to create later cell by cell the corresponding output images.

To determine the number of vessels contacts with the edges of the corresponding cell and the number of connected components within it, it is created the network skeleton of one pixel thick. The isolated pixels that do not make up a blood vessel are eliminated.

To keep control of results during the analysis of each cell, a State Matrix of $m \times n$ integer elements is created, where $m$ is the number of cells of height and $n$ is the number of cells of width of the segmented image. The values stored in the array (matrix) will indicate the status of the analysis of each cell according to their position in the image. This array will provide the necessary information for the separation algorithm to recognize the situation about all cells of the segmented vascular network being analyzed. Empty cells without any blood vessel inside it (zero in the matrix), 
obviously will be the last ones to be analyzed. A cell could be considered completely classified, when the analysis of it connection in all directions $(\mathrm{N}, \mathrm{S}, \mathrm{W}$ and $\mathrm{E})$ has been completed.

The cells around (or near to) the optic disc whose vessels already have been classified correctly by the user, either as venous or as arterial, before to initiate the separation of the vessel sub-networks, are taken as the baseline. From these initial cells a tree structure will be created taking it as the root.

The tree indicates only the order in which the cells are analyzed sequentially according to the path of blood vessels inside it. In each cell to be analyzed, not only are taken into account the characteristics of the vessels contained therein, but also the information inside of the adjacent cells in all directions. The end of each one of the shafts is determined by cells containing endpoint which obviously no longer generate children.

As the tree is traversed, the characteristics or parameters from each cell are obtained to determine the structure and type(s) of vessels contained inside it. For this, it is necessary to determine previously the following features and data of each cell being analyzed:

- The number of contacts between the skeletonized vessels and the cell edges.

- The number of connected components inside the cell.

- Analyze each one of the connected components in the cell and with the aid of the skeleton determine if it is a bifurcation, trifurcation, crossing point, ending point, or simply a vessel segment.

- Measure the thickness of the each blood vessel touching the edges of the cell with coordinates $(\mathrm{X}, \mathrm{Y})$ being analyzed and store the data together with the coordinates $(\mathrm{x}, \mathrm{y})$ where any pixel of the skeletonized blood vessel contacts with the edge.

To carry out the entire process of classification of vessels contained in each cell, a decision tree must be generated with the necessary rules to be taken into account from the contents of each cell and those adjacent to it, in order to classify them as arterial or venous. These rules are the following:

- To differentiate a crossing point from a bifurcation.

- To detect the type of particular points of the segmented network.

- If there is a branch which by their nature or position cannot be classified when it outs the optic disc, simply it is classified as indefinite and the white color is assigned to it.

- Crosses are always between two branches of different type; the type of each vessel depends on the type of the vessel previously classified according to the direction that it comes from.

Based on the parameters setting described previously and the cells already labeled that are located around the cell under analysis, the task is to classify this cell in one of possible combinations depending on the number of contacts with their four edges and on the structure of blood vessels located within it. This procedure is repeated with each one of the cells included in the table of cells not classified yet and those cells which still remains as partially classified, until all cells of the segmented image are completely classified. 


\section{Experimental Results and Discussion}

To evaluate the effectiveness of the module for separating the vascular networks, a total of 52 healthy fundus images were analyzed with respect to pathologies related to the blood vessels of the vascular network. From the 52 fundus images 26 proceed from the public DRIVE database [9] [10] and the other 26 images from the ARIA database [11].

Figures 2-12 show the resulting images after applying to an arbitrary image the proposed methodology to separate the venous and arterial networks. In Fig. 13 we can appreciate the vascular network manually separated in its venous and arterial branches. The metrics used to evaluate the quality of the separation of the vascular venous and arterial networks are the Recall, the Precision and the Coverage.

Fig. 2. Color fundus image




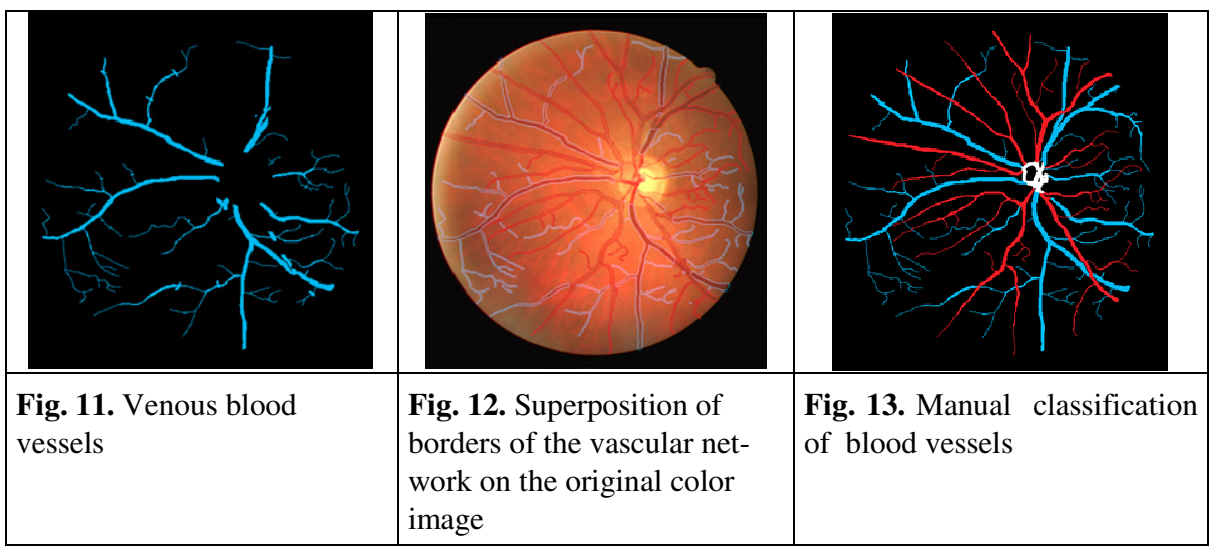

The methodology was applied to the 52 test images, after which the quality of the separation of each network was analyzed, first from the arterial network and later from the venous network. Firstly, in each case, a counting of the number of pixels was done in the network manually classified of each type (venous and arterial) that belongs to each them, correspondingly; this parameter was called total of the sample; later counting was done in both resulting images after the separation, both correctly and incorrectly classified and also the number of those pixels that were not classified by the proposed methodology. From these data the values of Recall, Precision and Coverage were calculated for both venous and arterial network independently. Finally, average values of these parameters were calculated for the 52 test images. Resulting values are shown in Table 1.

Table 1. Results from the evaluation of the classification quality

\begin{tabular}{|c|c|c|c|}
\hline \multirow{2}{*}{ Blood vessel type } & \multicolumn{3}{|c|}{ Measures } \\
\cline { 2 - 4 } & Recall (\%) & Precision (\%) & Coverage (\%) \\
\hline Arterials & 84.88 & 81.51 & 104.13 \\
\hline Venous & 82.87 & 86.63 & 95.66 \\
\hline Entire vascular network & 83.80 & 84.16 & 99.57 \\
\hline
\end{tabular}

As it can be noted from Table 1, acceptable percent was obtained in the quality of the separation by the proposed methodology, all of them above of the $80 \%$ measured on the base of pixel counting. For arterial blood vessels we get a classification capability of $84.88 \%$ while for venous blood vessels was of $82.87 \%$. This indicates us that the methodology classified correctly (as average) the $83.80 \%$ of the total blood vessels in the images. The value obtained for the Precision indicates that, as average, the proposed methodology was able to classify correctly the $84.16 \%$ from all blood vessels classified. Finally, the coverage of $104.13 \%$ obtained in arterial vessels is due to the reduction of the coverage obtained in the classification of venous vessels that result with an average coverage of $95.66 \%$. As a conclusion, from all images analyzed the methodology was able to classify more than $99 \%$ of all pixels of the original 
networks. Likewise, Table 2 tabulates the average error in percent calculated comparing the images with both networks manually separated in the original image and images with the venous and arterial blood vessels separated by our methodology.

Table 2. Average error (in percent) obtained in vascular network classification

\begin{tabular}{|c|c|}
\hline Blood vessel type & Average error (\%) \\
\hline Arterial & 18.49 \\
\hline Venous & 13.37 \\
\hline Entire vascular network & 15.84 \\
\hline
\end{tabular}

Another way to validate the quality of the separation of venous and arterial blood vessel networks is comparing the number of bifurcations and ending points, venous and arterials, as well as the crosses detected between the branches of both networks with the proposed methodology and those obtained from the composite image manually classified. As it can be noted the methodology was able to detect correctly, as average, $89.80 \%$ of crosses, $86.07 \%$ of bifurcations and $95.24 \%$ of ending points, resulting finally a global average detection of approximately $90 \%$. Also, the methodology was evaluated with respect to the invariance to the affine transformations like translation, rotation and scale. These tests were carried out over 10 images from the test set of the DRIVE database, to which small variations were applied in each case. After the application of small variations to the affine transformations of translation, rotation and changes in scale, the results obtained do not vary appreciably with respect to those obtained in images without such transformations, inclusive the results are a little better probably because the test sample set is smaller than in all previous cases. Then, we can conclude that the proposed methodology is invariant to the affine transformations of translation, rotation and change in scale. This means that we can use the proposed procedure to separate the venous and arterial networks over different images of the same eye of the same person. In all cases the results will be similar.

\section{Conclusions}

In this paper it has been demonstrated that the retina vascular network can be separated in its venous and arterial branches following the logical rules used for assembling a puzzle. The procedure is based on the physiological properties of the human retina vascular networks and the morphological properties of a tree structure as well. For arterial blood vessels we get a classification capability, based on the pixel counting, of $84.88 \%$ while for venous blood vessels was of $82.87 \%$. This indicates us that the methodology classified correctly (as average) the $83.80 \%$ of the total blood vessels in the images. The methodology was able to detect correctly, as average, $89.80 \%$ of crosses, $86.07 \%$ of bifurcations and $95.24 \%$ of ending points, resulting finally a global average detection of approximately $90 \%$. The semi-supervised methodology was evaluated also with respect to the invariance to the affine transformations of translation, rotation and scale. These tests were carried out over 10 images from the 
test set of the DRIVE database, to which small variations were applied in each case. In the case of translation, for the entire vascular network a Recall of $87.69 \%$, a Precision of $88.79 \%$, Coverage of 98.76 , and an error of $11.21 \%$ was obtained. In the case of the scale, corresponding values were $85.22 \%, 85.44 \%, 99.74 \%$ and $14.56 \%$. Finally, for rotation they were, correspondingly, $85.45 \%, 86.49 \%, 98.80 \%$ and $13.51 \%$. It is important to point out that the number of errors appears higher because those venous pixels located in cells where crosses were detected they were counted always as arterial ones. In the near future we will refine the error calculation when taking into consideration the venous pixels in all crosses detected.

\section{References}

1. Lei, T., Udupa, J.K., Saha, P.K., Odhner, D.: Artery-Vein separation via MRA- An image processing approach. IEEE Transactions on Medical Imaging 20(8), 689-703 (2001)

2. Rosa-Hernández, A., Olmedo-Maldonado, A., Hernández-Pérez, D., Felipe-Riverón, E.M., Sánchez-Garfias, F.A.: Separación y extracción de características de las redes vas-culares venosa y arterial en imágenes de retinas humanas.Tesis de licenciatura. IPN - Es-cuela Superior de Cómputo, México (2006)

3. Skands, U., Grunkin, M., Hansen, M.E.: Analysis of Fundus Images. United States Patent. US 6,996,260 B1 (2006)

4. Tek, H.: Method for artery-vein image separation in blood pool contrast agents. United States Patent. US2007/0249912A1 (2007)

5. Rothaus, K., Jiang, X., Rhiem, P.: Separation of the retinal vascular graph based upon structural knowledge. Image and Vision Computing (2008), doi:10.1016/j.mavis.2008.02.013

6. Niemeijer, M., van Ginneken, B., Abramoff, M.D.: Automatic classification of retinal vessels into arteries and veins. In: Medical Imaging 2009: Computer-Aided Diagnosis Proceedings of the SPIE, vol. 7620, pp. 7260IF-7260IF-8 (2009)

7. Tramontan, L., Ruggeri, A.: Computer estimation of the AVR parameter in diabetic retinopathy. In: WC 2009, IFMBE Proceedings 25/XI, pp. 141-144. Springer, Heidelberg (2009)

8. Muramatsu, C., Hatanaka, Y., Iwase, T., Hara, T., Fujita, H.: Automated selection of major arteries and veins for measurement of arteriolar-to-venular diameter ratio on retinal fundus images. Computerized Medical Imaging and Graphics 35, 472-480 (2011)

9. http://www.isi.uu.nl/Research/Datadase/DRIVE

10. Staal, J.J., Abramoff, M.D., Niemeijer, M., Viergever, M.A., van Ginneken, B.: Ridge based vessel segmentation in color images of the retina. IEEE Transactions on Medical Imaging 23, 501-509 (2004)

11. Farnell, D.: ARIA Online: Retinal Image Archive. St Paul's Eye Unit, Royal Liv-erpool University Hospital Trust, and Ophthalmology Clinical Sciences. University of Liverpool (2006), http: / / www. eyecharity.com/aria_online/

12. Villalobos-Castaldi, F.M., Felipe-Riverón, E.M., Albortante-Morato, C.: Segmenting Blood Vessels in Retinal Images Using an Entropic Thresh-olding Scheme. Advances in Computer Science and Engineering 42, 153-165 (2009) ISSN: 1870-4069

13. Martinez-Perez, M., Hughes, A., Stanton, A., Thom, S., Chapman, N., Bharath, A., Parker, K.: Retinal vascular tree morphology: A semi-automatic quantification. IEEE Transactions on Biomedical Engineering 49(8), 912-917 (2002) 